\title{
Daptomycin in bone and joint infections: a review of the literature
}

\author{
Dennis A. K. Rice $\cdot$ Luke Mendez-Vigo
}

Received: 28 August 2008 / Published online: 7 November 2008

(C) The Author(s) 2008. This article is published with open access at Springerlink.com

\begin{abstract}
Introduction To review the pharmacology, pharmacokinetics, efficacy, and safety of daptomycin, a novel antibiotic for the treatment of bone and joint infections, a literature search of relevant articles was conducted.

Materials and methods A PubMed/MEDLINE search (1990-April 2008) to identify relevant English-language literature was conducted. Search terms included bone and joint infection, osteomyelitis, daptomycin, and methicillinresistant Staphylococcus aureus (MRSA). Additional articles were identified by reviewing the bibliographies of articles cited. Programs and abstracts from infectious disease meetings were searched, and prescribing information of antibiotics indicated for bone and joint infections consulted. All articles identified from data sources published in English were evaluated.

Results Caused primarily by Gram-positive pathogens such as S. aureus and, to a lesser extent, Enterococcus faecalis, bone and joint infections are difficult to treat successfully. Surgical intervention and prolonged courses of antibiotics are frequently required, and failure of first-line antibiotic therapy is common. The emergence of $S$. aureus strains with reduced susceptibility to vancomycin, the longstanding gold standard for bone and joint infections, has
\end{abstract}

D. A. K. Rice

St. Joseph's/Candler Health System, Savannah, GA, USA

Present Address:

D. A. K. Rice

Ortho-McNeil Janssen, LLC, Fayetteville, GA, USA

L. Mendez-Vigo $(\bowtie)$

Cubist Pharmaceuticals, Inc., 65 Hayden Avenue,

Lexington, MA 02421, USA

e-mail: Luke.Mendez-Vigo@Cubist.com complicated the clinical scenario. Few randomized trials comparing the efficacy of different antibiotics for bone and joint infections exist. Daptomycin, a novel intravenous lipopeptide antibiotic, has shown potent in vitro activity against a broad spectrum of Gram-positive bacteria, including many resistant pathogens commonly associated with bone and joint infections such as MRSA and vancomycinresistant E. faecalis. Early clinical investigation of daptomycin in bone and joint infections unresponsive to antibiotics, such as vancomycin, has found a cure rate of approximately $80 \%$, with a low incidence of adverse events and drug resistance.

Conclusion Further studies are warranted to determine if limited clinical evidence, described in individual case reports and a daptomycin-specific retrospective registry, suggests daptomycin is a promising option for patients with bone and joint infections such as MRSA osteomyelitis.

Keywords Bone $\cdot$ Daptomycin $\cdot$ Infection ·

Osteomyelitis $\cdot$ MRSA $\cdot$ VRE

\section{Introduction}

The treatment of bone and joint infections such as osteomyelitis, septic arthritis, and prosthetic joint infections can be difficult, requiring prolonged administration of antibiotics and extensive surgical procedures [1]. Most bone and joint infections are caused by Gram-positive cocci including Staphylococcus spp. and enterococci [1], increasingly resistant to commonly used antibiotics such as methicillin and other $\beta$-lactam agents [2,3]. Vancomycin is often used to treat bone and joint infections caused by methicillin-resistant Staphylococcus aureus (MRSA), an organism seen with increasing frequency [4]. MRSA has recently emerged 
as an important cause of community-acquired infection in many parts of the United States [5], and recent surveys of S. aureus isolates report MRSA rates as high as $74 \%[6,7]$. Although vancomycin remains a first-line treatment for many bone and joint infections caused by typical organisms, its efficacy against MRSA infections in other settings is declining ominously [8]. This reduced susceptibility of S. aureus to vancomycin [3, 9] may be partially due to S. aureus-produced biofilms, which may facilitate resistance by promoting horizontal gene transfer [10-12]. The ability of pathogens to escape lysis and killing by vancomycin, termed "tolerance," represents an additional risk. Both genetic and physiologic factors can selectively suppress susceptibility to the killing action of antibiotics without a change in the minimum inhibitory concentration (MIC), i.e., can make bacteria antibiotic-tolerant and difficult to detect using standard susceptibility testing methods $[13,14]$.

\section{Selection of antimicrobial therapy for bone and joint infections}

Most cases of osteomyelitis require antimicrobial therapy, adequate drainage, thorough debridement, obliteration of dead space, and wound protection. Patients are initially treated empirically with broad-spectrum antimicrobials, changed to targeted antimicrobial therapy based on bone culture results of samples taken at debridement surgery or from deep bone biopsies. Septic arthritis, a medical emergency, requires prompt recognition and aggressive treatment for a good prognosis [15]. Delays in surgical drainage and antibiotic therapy may lead to progressive synovitis and irreversible destruction of cartilage and bone [16, 17]. Empirical antibiotic therapy directed against staphylococci, the most common causative organisms of septic arthritis in adults, should be given while awaiting culture results $[15,18]$. If the Gram stain is negative in acute septic arthritis, empirical therapy should include an antimicrobial having activity against $S$. aureus and streptococci $[15,19]$. Antibiotic therapy of prosthetic joint infections is based on a definitive microbiologic diagnosis, usually after the surgical removal of all bioprosthetic components [15].

Despite these general guidelines, high-quality evidence of the relative effectiveness of various regimens of antibiotic therapy, and their optimal duration, is scant [20]. A 2001 meta-analysis concluded that little high-quality evidence-based therapy for osteomyelitis and septic arthritis exists [21]. Increasing resistance to antibiotics has prompted the development of new drugs to treat Grampositive infections, which may also be used in the treatment of bone and joint infections.

\section{Current treatment options for $S$. aureus bone and joint infections}

Staphylococcus aureus is the most common organism implicated in osteomyelitis and septic arthritis [1, 15]. Although the term MRSA denotes resistance to methicillin, it is synonymous with multidrug-resistant $S$. aureus, because many nosocomial MRSA strains are resistant to many other commonly used antibiotics. Approximately $20 \%$ of S. aureus isolates in Europe are reported as methicillin-resistant; the prevalence ranges from 33 to $55 \%$ in US hospitals. Since 1996, seven vancomycin-intermediate S. aureus (VISA) strains (i.e., vancomycin MIC $=8-16 \mathrm{mg} / \mathrm{L}$ ) have been identified in Europe, Asia, and the United States, and VISA strains (vancomycin MIC $\geq 32 \mathrm{mg} / \mathrm{L}$ ) were also reported in the United States between 2002 and 2005 [22].

Vancomycin was the last available drug to which $S$. aureus had remained uniformly sensitive, until recent reports of low-level glycopeptide resistance and the transfer of high-level vancomycin resistance from Enterococcus to $S$. aureus $[23,24]$. A limited number of broad-spectrum antimicrobials are available to combat multidrug-resistant Gram-positive organisms.

Vancomycin

In 1997, the first clinical isolate of $S$. aureus having intermediate resistance to vancomycin was reported from Japan [25]. Since then, many $S$. aureus isolates with reduced susceptibility to glycopeptides have been reported from various locales $[23,26]$. The first report of vancomycinresistant $S$. aureus in the United States occurred in 2002. The patient had been treated with multiple antibiotics, including vancomycin. MRSA bacteremia later developed, and the patient was treated with vancomycin and rifampin. The Centers for Disease Control and Prevention confirmed that the $S$. aureus isolated was resistant to oxacillin, vancomycin, and teicoplanin [3].

Vancomycin itself has poor bone penetration and in some animal studies has shown an inability to sterilize bone $[27,28]$. When bone and joint pathogens are susceptible, vancomycin concentrations in serum must be monitored, and vancomycin-associated adverse events (e.g., marrow suppression, ototoxicity, nephrotoxicity, and rash) may discourage its use [29]. Adverse effects, increasing MICs, and the increased prevalence of vancomycin-resistant enterococci (VRE), VISA, and vancomycin-resistant $S$. aureus (VRSA) are already limiting the use of vancomycin in the treatment of bone and joint infections [23, 26, 30, 31]. Vancomycin-susceptible clinical MRSA isolates demonstrate considerable heterogeneity in vitro with respect to vancomycin MIC and vancomycin killing, affecting the clinical 
efficacy of the drug. A significant risk for vancomycin treatment failure in MRSA bacteremia has been demonstrated, with increasing vancomycin MICs still well within the susceptible range [32]. An increased risk of recurrence seen with vancomycin treatment of S. aureus osteomyelitis [33] further diminishes the clinical utility of this agent for the treatment of bone and joint infections.

\section{Quinupristin/dalfopristin}

Quinupristin/dalfopristin (Q-D), the first parenteral streptogramin, achieves a response in approximately two-thirds of patients with MRSA infections. This combination product also has efficacy in patients with Gram-positive complicated skin and skin structure infections (cSSIs) and nosocomial pneumonia [34, 35]. Rifampin plus either Q-D or vancomycin has been found significantly more effective than monotherapy in a preclinical trial of MRSA knee infection [36].

An evaluation of Q-D for the treatment of a variety of MRSA infections (44\% bone and joint infections) in patients either intolerant of or failing prior therapy resulted in an overall success rate of $71.1 \%$ in the all-treated population $(n=90)$ and $66.7 \%$ in patients who were both clinically and bacteriologically evaluable $(n=27)$. The most common non-venous adverse events were arthralgias (10.8\%), myalgias (8.6\%), and nausea (8.6\%) [37]. Q-D is not indicated for bone and joint infections, and little clinical data exist for the drug's use in that setting [34].

\section{Linezolid}

Linezolid is an oxazolidinone, a new class of antibacterial agents particularly effective against Gram-positive infections, including methicillin- and vancomycin-resistant strains [38]. It is available in oral and intravenous (IV) formulations and has been approved by the Food and Drug Administration for the treatment of numerous infections, including cSSIs or uncomplicated SSIs, without concomitant osteomyelitis, caused by S. aureus [39]. However, data on the effectiveness and tolerability of linezolid as prolonged therapy for bone and joint infections are lacking, and linezolid is not indicated for their treatment. No large, randomized trials have been published on the use of linezolid for orthopedic infections such as osteomyelitis and septic arthritis. Nevertheless, a recent retrospective study of linezolid for chronic osteomyelitis found that when used as monotherapy, or in combination with other antimicrobials and/or surgery, linezolid was associated with a cure rate of $85 \%$ at 12 weeks after the end of treatment and $78.8 \%$ at follow-up. Adverse events, including anemia and peripheral neuropathy, were reported in $51.5 \%$ of subjects, and $34.8 \%$ of subjects discontinued the study because of adverse events [40]. Peripheral and optic neuropathy have been reported in patients treated with linezolid, primarily in patients treated for longer than the maximum recommended duration of 28 days [39]. Early identification of linezolidinduced peripheral neuropathy is a particular concern, because this may be irreversible [41]. Resistance to linezolid has been reported among strains of both MRSA and Enterococcus faecium [37, 42, 43].

Tigecycline

Tigecycline, the first glycylcycline approved in the United States, is indicated for cSSIs and complicated intra-abdominal infections (cIAIs), not for bone and joint infections. Tigecycline has activity against both methicillin-susceptible S. aureus (MSSA) and MRSA. In four phase III trials in patients with cSSIs and cIAIs, tigecycline was noninferior to its comparators (vancomycin + aztreonam in two studies and imipenem/cilastatin in two studies), with clinical cure rates among clinically evaluable patients of $>80 \%$ $(P<0.001$ for noninferiority). Although there have been no human trials involving osteomyelitis, animal studies suggest tigecycline may have a role in treating bone infection. After 28 days of treatment of experimentally induced osteomyelitis, rabbits receiving tigecycline/oral rifampicin showed a $100 \%$ infection clearance [44]. Frequently reported adverse events include nausea and vomiting, diarrhea, local IV-site reaction, infection, and fever [45, 46]. Resistance to tigecycline has been reported among both Gram-positive and Gram-negative strains.

\section{Daptomycin}

This novel cyclic lipopeptide is bactericidal against Grampositive bacteria, including MRSA and VRE. Daptomycin kills Gram-positive bacteria by disruption of multiple bacterial plasma membrane functions, without penetrating the cytoplasm [47]. Insertion of the lipophilic daptomycin tail into the bacterial cell membrane causes rapid membrane depolarization and a potassium ion efflux. Arrest of DNA, RNA, toxin production, and protein synthesis follows, resulting in bacterial cell death without lysis of the cell wall (Fig. 1) [48-50].

\section{Microbiology}

The in vitro potency of daptomycin has been demonstrated against a wide range of aerobic and anaerobic Grampositive bacteria, including MRSA, glycopeptide-intermediate $S$. aureus, methicillin-resistant coagulase-negative staphylococci, and VRE [47]. The in vitro activity of daptomycin against three VISA isolates was compared with that of vancomycin, linezolid, and Q-D. Overall, daptomycin 


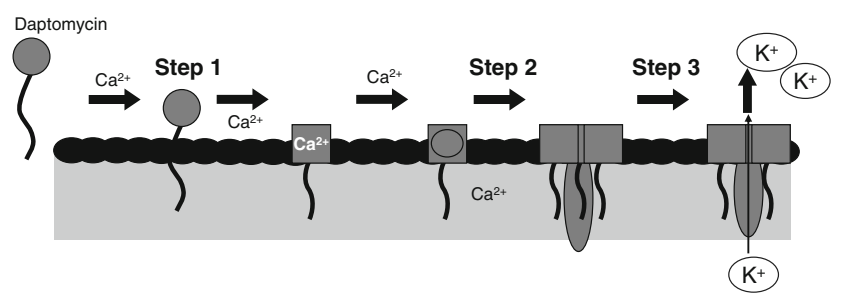

Fig. 1 Mechanism of action of daptomycin [48]. Reprinted with permission. Step 1 calcium-dependent binding and insertion of the lipophilic tail into Gram-positive cytoplasmic membrane. Step 2 oligomerization and channel formation occur. Step 3 ion leakage and collapse of organism lead to cell death

was more active against all organisms tested, except $E$. faecium and VISA, against which its activity was similar to that of Q-D. In time-kill studies with MRSA, MRSE, VRE, and VISA, daptomycin demonstrated greater bactericidal activity than all others tested, killing $\geq 3 \log \mathrm{CFU} / \mathrm{mL}$ within $8 \mathrm{~h}$ [51]. Daptomycin is also effective against a variety of streptococci, such as $\beta$-hemolytic streptococci and other Streptococcus spp. [52]. Susceptibility criteria are shown in Table 1. Synergy with daptomycin has been described in vitro with aminoglycosides, i.e., gentamicin, oxacillin, other $\beta$-lactams, and rifampicin [47, 53, 54]. Daptomycin exhibits a dose-dependent post-antibiotic effect lasting from 1 to $6 \mathrm{~h}$ against $E$. faecalis and $S$. aureus after exposure to concentrations ranging from 0.25 to $16 \mathrm{mg} / \mathrm{L}$ (i.e., between one- and eightfold the MIC) [55].

\section{Pharmacology}

With an 8-h half-life, once-daily dosing results in linear pharmacokinetics at doses up to $12 \mathrm{mg} / \mathrm{kg}$, with minimal drug accumulation (Table 2) [47]. Daptomycin distributes primarily in the plasma, with penetration to vascularized tissues. The drug is highly protein-bound (92\%); excretion occurs primarily via the kidneys. Approximately $80 \%$ of the total dose, of which two-thirds is intact drug, is recovered in the urine $[47,56]$. In patients with severe renal impairment (creatinine clearance $<30 \mathrm{~mL} / \mathrm{min}$ ), the dosing interval is increased from once daily to every $48 \mathrm{~h}$. Daptomycin's unique mechanism of action and its lack of metabolization by cytochrome $\mathrm{P} 450$ or other hepatic enzymes results in an absence of drug-drug interactions. Against 70 clinical isolates, interactions between daptomycin and 25 antimicrobials were additive or indifferent; antagonism was not observed. Synergistic interactions were observed between daptomycin and gentamicin against staphylococci and enterococci, including strains resistant to methicillin and vancomycin; in vitro synergistic interactions of daptomycin and rifampin have been shown against methicillin- and vancomycin-resistant staphylococci and enterococci $[47,56]$.

Skeletal muscle was the most sensitive tissue to the adverse effects of daptomycin in animal studies. Mild myopathy was easily predicted and monitored by measuring serum creatine phosphokinase concentrations; the effect was reversible upon the cessation of therapy $[47,56]$. Dog studies demonstrated that adverse effects increased with fractionated compared with once-daily administration of the same total daily dose. Thus, once-daily dosing may increase the therapeutic-toxicity ratio by increasing efficacy and decreasing adverse effects [57].

Preclinical efficacy of daptomycin in bone and joint infection models

The efficacy of daptomycin against a range of infections has been demonstrated in animal studies. Using a variety of antibiotic-resistant and antibiotic-sensitive Gram-positive bacteria, daptomycin eradicated infections in the blood, muscle, kidney, heart, and bone tissues of animals [57-64]. In a rat model of chronic MSSA osteomyelitis, daptomycin $10 \mathrm{mg} / \mathrm{kg}$ subcutaneously every $12 \mathrm{~h}$ resulted in bacterial numbers similar to those of untreated animals, a finding

Table 1 Daptomycin susceptibility criteria [47]

\begin{tabular}{|c|c|c|c|}
\hline \multirow[t]{2}{*}{ Pathogen } & \multicolumn{3}{|c|}{ Broth dilution $\mathrm{MIC}^{\mathrm{a}}(\mu \mathrm{g} / \mathrm{mL})$} \\
\hline & $\mathrm{S}$ & I & $\mathrm{R}$ \\
\hline Staphylococcus aureus (methicillin-susceptible and methicillin-resistant) & $\leq 1$ & $\mathrm{~b}$ & $\mathrm{~b}$ \\
\hline Streptococcus pyogenes, $S$. agalactiae, and $S$. dysgalactiae sub-spp. equisimilis & $\leq 1$ & $\mathrm{~b}$ & $\mathrm{~b}$ \\
\hline Enterococcus faecalis (vancomycin-susceptible only) & $\leq 4$ & $\mathrm{~b}$ & $\mathrm{~b}$ \\
\hline
\end{tabular}

$I$ intermediate, $R$ resistant, $S$ susceptible

a The mean inhibitory concentration (MIC) interpretive criteria for $S$. aureus and E. faecalis are applicable only to tests performed by broth dilution using Mueller-Hinton broth adjusted to a calcium content of $50 \mathrm{mg} / \mathrm{L}$; the MIC interpretive criteria for Streptococcus spp. other than S. pneumoniae are applicable only to tests performed by broth dilution using Mueller-Hinton broth adjusted to a calcium content of 50 mg/L, supplemented with 2-5\% lysed horse blood, inoculated with a direct colony suspension and incubated in ambient air at $35^{\circ} \mathrm{C}$ for $20-24 \mathrm{~h}$

b The current absence of data on daptomycin-resistant isolates precludes defining any categories other than "susceptible." Isolates yielding test results suggestive of a "non-susceptible" category should be retested, and if the result is confirmed, the isolate should be submitted to a reference laboratory for further testing 
Table 2 Pharmacokinetic parameters of daptomycin at steady-state in healthy subjects [47]

\begin{tabular}{llllll}
\hline Dose $(\mathrm{mg} / \mathrm{kg})^{\mathrm{a}}$ & \multicolumn{3}{l}{ Pharmacokinetic parameters } & & \\
\cline { 2 - 6 } & $\mathrm{AUC}_{0-24}(\mu \mathrm{g} \mathrm{h} / \mathrm{mL})$ & $t_{1 / 2}(\mathrm{~h})$ & $V_{\mathrm{ss}}(\mathrm{L} / \mathrm{kg})$ & $\mathrm{CL}_{\mathrm{T}}(\mathrm{mL} / \mathrm{h} \mathrm{per} \mathrm{kg})$ & \multicolumn{1}{l}{$C_{\mathrm{max}}(\mu \mathrm{g} / \mathrm{mL})$} \\
\hline $4(n=6)$ & $494(75)$ & $8.1(1.0)$ & $0.096(0.009)$ & $8.3(1.3)$ & $57.8(3.0)$ \\
$6(n=6)$ & $632(78)$ & $7.9(1.0)$ & $0.101(0.007)$ & $9.1(1.5)$ & $93.9(6.0)$ \\
$8(n=6)$ & $858(213)$ & $8.3(2.2)$ & $0.101(0.013)$ & $9.0(3.0)$ & $123.3(16.0)$ \\
$10(n=9)$ & $1,039(178)$ & $7.9(0.6)$ & $0.098(0.017)$ & $8.8(2.2)$ & $141.1(24.0)$ \\
$12(n=9)$ & $1,277(253)$ & $7.7(1.1)$ & $0.097(0.018)$ & $9.0(2.8)$ & $183.7(25.0)$ \\
\hline
\end{tabular}

$\mathrm{AUC}_{0-24}$, area under the concentration-time curve from 0 to $24 \mathrm{~h} ; t_{1 / 2}$, terminal elimination half-life; $V_{\mathrm{ss}}$, volume of distribution at steady-state; $\mathrm{CL}_{\mathrm{T}}$, plasma clearance; $C_{\max }$, maximum plasma concentration

${ }^{a}$ Doses of daptomycin $>6 \mathrm{mg} / \mathrm{kg}$ have not been approved

possibly related to underdosing [65]. Examination of several doses of daptomycin in an experimental murine S. aureus thigh infection model demonstrated concentration-dependent bactericidal activity. A mean peak/MIC ratio of 255 or a mean 24-h area under the concentrationtime curve (AUC)/MIC ratio of 1,061 was needed to effect a $2 \log _{10}$ reduction in vivo [66].

Daptomycin reduced bacterial cultures of bone infected with $S$. aureus in an experimental osteomyelitis rabbit model [63]. Daptomycin was as effective as vancomycin at comparable peak plasma levels of approximately $33 \mu \mathrm{g} / \mathrm{mL}$ for the two drugs [63]. High-dose daptomycin was compared with standard regimens of oxacillin and vancomycin in a difficultto-treat rat tissue cage model of chronic $S$. aureus foreign body infection, so called as perforated Teflon cylinders are implanted subcutaneously in rats, and infected, in this case with MSSA strain I20, a clinical isolate from catheter-related sepsis. In vitro elimination of strain I20 was more rapid with $8 \mathrm{mg} / \mathrm{L}$ of daptomycin than with oxacillin or vancomycin at the same dose. After 7 days of therapy, the mean $( \pm$ SEM) reduction in viable counts of MSSA I20 was 2.62 $( \pm 0.30) \log _{10} \mathrm{CFU} / \mathrm{mL}$ in cages $(n=18)$ of daptomycintreated rats, exceeding by more than twofold $(P<0.01)$ the viable count reductions in cages of oxacillin-treated and vancomycin-treated rats, respectively [67]. A comparative experiment of daptomycin and vancomycin in a rabbit model of MRSA osteomyelitis found that ten rabbits given daptomycin $25 \mathrm{mg} / \mathrm{kg}$ per day showed a $90 \%$ infection clearance, nine rabbits treated with daptomycin $15 \mathrm{mg} / \mathrm{kg}$ per day showed a $66.67 \%$ clearance, and eight rabbits treated with vancomycin showed a $33.3 \%$ clearance. Untreated controls $(n=15)$ demonstrated only a $13.3 \%$ clearance [68].

\section{Daptomycin in bone and joint infections}

Daptomycin was initially approved in 2003 for the treatment of cSSIs caused by $S$. aureus (including methicillinresistant strains), S. pyogenes, S. agalactiae, S. dysgalactiae sub-spp. equisimilis, and E. faecalis (vancomycin-susceptible strains only) at a dose of $4 \mathrm{mg} / \mathrm{kg}$ per day administered IV. More than $90 \%$ of vancomycin-resistant $E$. faecalis isolates have demonstrated an in vitro MIC $\leq$ the susceptible breakpoint for daptomycin, but the efficacy of daptomycin in treating clinical infections caused by these microorganisms has not been established in clinical trials [47]. In 2006, daptomycin was approved as a once-daily therapy $(6 \mathrm{mg} /$ $\mathrm{kg}$ ) for the treatment of $S$. aureus bloodstream infections (bacteremia), including right-sided endocarditis caused by MSSA and MRSA [47].

The use of daptomycin has been investigated in bone and joint infections. Case records of a prospective, randomized trial of daptomycin alone versus gentamicin plus either semisynthetic penicillin or vancomycin for $S$. aureus bacteremia and endocarditis [8] were retrospectively assessed to identify patients in the trial with concomitant complicated bone and joint infections (i.e., osteomyelitis or septic arthritis). Bone and joint infections were found in 21 of 120 patients treated with daptomycin $(17.5 \%)$ and 11 of 115 control patients $(9.6 \%)$. Treatment success was achieved in 16 patients treated with daptomycin by the end of therapy (76.2\%) and was maintained in 14 patients (66.7\%) 6 weeks after the end of therapy. Seven of 11 control patients achieved a successful outcome by the end of therapy $(63.6 \%)$, with 6 patients $(54.6 \%)$ maintaining success 6 weeks later [69].

A second retrospective chart review of patients with osteomyelitis treated with daptomycin was conducted at six sites in the United States. Anatomic sites of infection included the lower extremity (45\%), upper extremity (24\%), vertebrae (21\%), and sternum/chest wall $(10 \%)$; $41 \%$ of patients had prosthetic material present. The most common pathogen isolated was MRSA (64\%). Fifty-two percent of patients received an antibiotic within 4 weeks of daptomycin treatment (vancomycin in $67 \%$ of cases). Forty-five percent of patients started daptomycin because previous therapy had failed. The median daptomycin dosage was $4.2 \mathrm{mg} / \mathrm{kg}$ (range $3.5-7.1 \mathrm{mg} / \mathrm{kg}$ ), and the median 
duration of therapy was 42 days (range 18-63 days). Fortyeight percent of patients received an antibiotic along with daptomycin, most commonly rifampin. Outcomes at end of therapy were cure $(16 / 29,55 \%)$, improved $(10 / 29,34 \%)$, and failure $(3 / 29,10 \%)$. Outcomes at follow-up were cure $(19 / 29,65 \%)$, improved $(1 / 29,3 \%)$, and failure $(9 / 29$, $31 \%$ ). Clinical success (i.e., cure plus improved) was achieved with daptomycin in $90 \%$ of patients with osteomyelitis by the end of therapy, despite the large percentage who had failed treatment with prior antibiotics. Clinical success was maintained in $69 \%$ of patients at $\geq 6$ months' follow-up. During the study period, there were six adverse events reported in six patients, all mild to moderate in severity [70].

Three case series on the use of daptomycin in bone and joint infections included 22 male and 31 female patients with a mean age of 65.8 years [33, 41, 71, 72]. Osteomyelitis was diagnosed in $43 \%$ of patients; other infections included total joint arthroplasty, laminectomy site infection, knee abscess, and bacteremia associated with septic arthritis. MRSA was isolated as the pathogen in $74 \%$ of patients, and more than one pathogen was isolated in $11 \%$ of cases. Daptomycin was given at a dosage of $4-6 \mathrm{mg} / \mathrm{kg}$ per day for a mean duration of 37.4 days. Patients were previously treated with antibiotics such as vancomycin, rifampicin, ciprofloxacin, linezolid, and Q-D. Cure of infection was achieved with daptomycin in $81 \%$ of cases, with a cure rate of $100 \%$ among patients with osteomyelitis and $60 \%$ of total joint arthroplasty infection cases. Development of non-susceptibility to daptomycin occurred in one case of MRSA infection of an epidural abscess [33]. A summary of ten patients from one case series who were treated with daptomycin for various bone and joint infections is shown in Table 3. Whether the effectiveness of treatment of bone and joint infection in each of these three case series can be solely attributed to daptomycin is uncertain, since other antibiotics were co-administered in most of the cases [41]; however, as previously stated, most of these patients had been treated with other antibiotics that did not result in a positive outcome, necessitating the change to daptomycin.

The outcomes of 25 patients with foot or ankle osteomyelitis who were treated with daptomycin at a median dose of $6 \mathrm{mg} / \mathrm{kg}$ (range $4-6.2 \mathrm{mg} / \mathrm{kg}$ ) for a median duration of 38 days (range 6-59 days) were recently reported [73]. Twenty-three patients received daptomycin as secondary or tertiary therapy, primarily because they had not responded to prior antibiotic treatment $(n=15)$. Concomitant antibiotics were given to 11 patients, mostly for Gram-negative and/or anaerobic coverage. MRSA was the most common pathogen overall (15 of 25 patients). At the end of therapy, 16 patients' symptoms had resolved, eight patients improved, and one patient did not respond to therapy. At a median follow-up of 9 weeks, 19 patients' symptoms resolved, three patients improved, and three patients did not respond to therapy. Additional antibiotics were given to $52 \%$ of patients for a minimum of 8 days (median 30 days, range 8-232 days). Four patients with an implant (all removed) were successfully treated. Daptomycin appeared to be effective for previously treated foot and ankle osteomyelitis caused by Gram-positive bacteria in this trial, warranting additional controlled clinical studies of daptomycin as an alternative agent for patients with bone and joint

Table 3 Case summary of patients with bone and joint infections treated with daptomycin [33]

\begin{tabular}{|c|c|c|c|c|c|c|}
\hline Patient number & Age/gender & Diagnosis & Pathogen & Prior antibiotic & DAP duration (days) & Resolution $^{\mathrm{a}}$ \\
\hline \multicolumn{7}{|c|}{ Rush Presbyterian St. Luke's Medical Center } \\
\hline 1 & $47 / \mathrm{F}$ & Osteo & MRSA & VAN & 8 & Yes \\
\hline 2 & $41 / \mathrm{M}$ & Septic joint, bact & MRSA & None & 44 & Yes \\
\hline 3 & $87 / \mathrm{F}$ & Septic arthritis, bact & MRSA & VAN, GEN & 41 & Relapse \\
\hline 4 & $67 / \mathrm{F}$ & Septic joint & Entero & VAN & 4 & Yes (w/amp) ${ }^{\mathrm{b}}$ \\
\hline \multicolumn{7}{|c|}{ Fountain Valley Regional Hospital } \\
\hline 5 & $58 / \mathrm{F}$ & Septic arthritis, osteo, bact & MRSA & VAN, LIN, QUI & 42 & Yes \\
\hline 6 & $57 / \mathrm{F}$ & Osteo, wound infection & MRSA & VAN & 35 & Yes \\
\hline 7 & $83 / \mathrm{F}$ & Osteo & MRSA & VAN, QUI, LIN, MIN/RIF & 28 & Yes \\
\hline 8 & $72 / \mathrm{M}$ & Osteo & MRSA & VAN & 21 & Yes \\
\hline 9 & $67 / F$ & Osteo, septic arthritis & MRSA & VAN, QUI, CEF, LIN & 28 & Yes \\
\hline 10 & $39 / \mathrm{F}$ & Osteo & Strep & $\mathrm{CEF}$ & 42 & Yes \\
\hline
\end{tabular}

Reprinted with permission

Osteo osteomyelitis, bact bacteremia, MRSA methicillin-resistant Staphylococcus aureus, Entero Enterococcus faecalis, Strep presumed streptococcus infection (no cultures available), VAN vancomycin, GEN gentamicin, LIN linezolid, QUI quinupristin/dalfopristin, MIN/RIF minocycline/ rifampin, $C E F$ cefepime, $D A P$ daptomycin

${ }^{a}$ Resolution of signs and symptoms associated with the infection and discharge from the hospital

b Patient was switched to ampicillin once the pathogen was identified and was successfully treated 
infections who are intolerant to other antibiotics or fail to improve. In a recent case report, continual daptomycin use for 18 months in a patient with MRSA osteomyelitis is noteworthy because of the extended duration of daptomycin use without significant toxicity and because of ultimate efficacy in treating the infection [74]. To date, no randomized, controlled trials comparing the effectiveness and safety of daptomycin with other antibiotics used to treat bone and joint infections have been completed [41].

The Cubicin Outcomes Registry and Experience (CORE) is a multicenter retrospective observational database containing information about patients treated with daptomycin in clinical practice. The characteristics and outcomes of patients with osteomyelitis who were treated with daptomycin were retrospectively evaluated from the CORE 2004 database [75]. Of the 67 patients with osteomyelitis who were clinically evaluable at the end of daptomycin therapy, 42 (63\%) were cured, 13 (19\%) were improved, $7(10 \%)$ had failure, and $5(7 \%)$ were nonevaluable. In about one half of the cases $(48 \%)$, daptomycin was given concurrently with other antimicrobial agents; MRSA comprised $45 \%$ of the pathogens. Success occurred at a $94 \%$ rate when daptomycin was used alone, with no antimicrobial agents following it. These results indicate that daptomycin can be effectively used in clinical practice to treat osteomyelitis caused by a Gram-positive organism, including MRSA [75].

\section{Antibiotic-loaded polymethlymethacrylate bone cement}

Polymethlymethacrylate (PMMA) bone-cement spacers have been used in the revision of infected prosthetic joints and in the placement of orthopedic implants in patients at high risk for infection. The ability to deliver high local concentrations of antimicrobial agents with PMMA has made it a standard of care for patients with chronic infection at the site of a total joint replacement. The most commonly used antibiotics include tobramycin, gentamicin, vancomycin, and cephalosporins [76, 77].

A Mayo Clinic study recently evaluated the elution of daptomycin from PMMA in a continuous flow chamber designed to simulate in vivo conditions. Three-millimeter beads containing $2.5,7.5$, and $15.0 \%$ daptomycin (weight daptomycin per weight PMMA) were individually placed in a chamber with $1 \mathrm{~mL}$ Krebs Ringer buffer flowing at $1 \mathrm{~mL} / \mathrm{h}$. The majority of daptomycin was released in the first $24 \mathrm{~h}$. When treating osteomyelitis, achieving the highest local concentration of antimicrobial is important. In this study, daptomycin eluted from 3-mm PMMA beads in a continuous flow chamber more completely than did vancomycin when tested at the same laboratory [78]. By doubling the amount of daptomycin in PMMA, local peak concentra- tions of drug were significantly increased [77]. In a recent study in which daptomycin was mixed with calcium sulfate hemihydrate to form a hardened pellet, daptomycin eluted from calcium sulfate for up to 28 days retained the ability to inhibit growth of $S$. aureus and S. epidermidis [79].

\section{Resistance to daptomycin}

As with all antibiotics, orthopedic cases of resistance to daptomycin have been reported, including patients with vertebral or sternal osteomyelitis, diskitis, and septic arthritis [80-82], all positive for MRSA. Interestingly, patients in all four reports had received previous vancomycin treatment. A positive correlation between reduced daptomycin susceptibility and vancomycin resistance in VISA has been reported. Similar to the mechanism of vancomycin resistance, the physical barrier of a thickened cell wall may contribute to $S$. aureus resistance to daptomycin [83]. However, a definitive mechanism of resistance to daptomycin has not yet been identified, and there are no known transferable elements conferring resistance to the drug. The isolation of glycopeptide-intermediate S. aureus (GISA) is rare (i.e., $\leq 0.3 \%$ ) [84], and cross-resistance of daptomycin with vancomycin is seen in only a fraction of GISA isolates. Of 15 GISA isolates, $86.7 \%$ were susceptible to daptomycin, as reported in the US SENTRY Antimicrobial Surveillance Program [85]. Among almost 10,000 isolates tested, only four $(0.04 \%)$ had a daptomycin MIC of $2 \mu \mathrm{g} /$ $\mathrm{mL}$ (all were glycopeptide-susceptible $S$. aureus); only two $(0.02 \%)$ GISA isolates were found (vancomycin MIC $4 \mu \mathrm{g} /$ $\mathrm{mL}$; daptomycin MIC 0.5-1.0 $\mu \mathrm{g} / \mathrm{mL})$ [86, 87].

To date, daptomycin has been largely used as salvage therapy following vancomycin failure [88, 89]. In the study by Fowler et al. [8], most patients had received vancomycin therapy for a mean of 2 days before starting either daptomycin or standard therapy. A post hoc analysis of MRSAinfected patients from this study reported that previous vancomycin therapy did not affect the clinical outcome of daptomycin-treated patients [90, 91]. Nonetheless, the activity of daptomycin against MRSA may be best exploited in patients who are treated with daptomycin early; heavy exposure to vancomycin prior to treatment with daptomycin may increase the likelihood of a suboptimal response [92].

\section{Summary}

Gram-positive organisms, particularly $S$. aureus, are responsible for the majority of bone and joint infections. The prevalence of increasingly resistant organisms is a major concern, both for achieving therapeutic success and 
because of broader cross-resistance implications [27]. While vancomycin has long been the gold standard for the treatment of bone and joint infections, the emergence of glycopeptide tolerance and resistance to vancomycin, resulting in clinical failures [23, 26, 30, 31], demonstrate the need for alternative treatments.

Daptomycin has exhibited activity in the treatment of Gram-positive bone and joint infections, including those caused by MRSA and VRE [93]. It is rapidly bactericidal, with reported $\mathrm{MIC}_{90}$ concentrations against MRSA and coagulase-negative staphylococci at $0.5 \mu \mathrm{g} / \mathrm{mL}$ [94]. Daptomycin appears effective against multidrug-resistant Gram-positive pathogens commonly found in osteomyelitis and joint infections, even when other first-line antibacterial treatments have failed [41]. Daptomycin is well tolerated, with a low potential for adverse events, and the risk of spontaneous resistance appears low [49, 83]. The novel mode of action, rapid in vitro bactericidal activity against growing and stationary-phase bacteria, a once-daily dosing regimen, and no requirement for drug monitoring contribute to its potential therapeutic utility [50].

Open Access This article is distributed under the terms of the Creative Commons Attribution Noncommercial License which permits any noncommercial use, distribution, and reproduction in any medium, provided the original author(s) and source are credited.

\section{References}

1. Davis JS (2005) Management of bone and joint infections due to Staphylococcus aureus. Intern Med J 35(suppl 2):S79-S96. doi:10.1111/j.1444-0903.2005.00982.x

2. Razonable RR, Osmon DR, Steckelberg JM (2004) Linezolid therapy for orthopedic infections. Mayo Clin Proc 79:1137-1144

3. Sievert DM, Boulton ML, Stoltman G et al (2002) Staphylococcus aureus resistant to vancomycin-United States 2002. Morb Mortal Wkly Rep 51:565-567

4. Rice LB (2006) Antimicrobial resistance in Gram-positive bacteria. Am J Infect Control 34(suppl 5):S11-S19. doi:10.1016/ j.ajic. 2006.05 .220

5. Tenover FC, McDougal LK, Goering RV et al (2006) Characterization of a strain of community-associated methicillin-resistant Staphylococcus aureus widely disseminated in the United States. J Clin Microbiol 44:108-118. doi:10.1128/JCM.44.1.108118.2006

6. Styers D, Sheehan DJ, Hogan P, Sahm DF (2006) Laboratorybased surveillance of current antimicrobial resistance patterns and trends among Staphylococcus aureus: 2005 status in the United States. Ann Clin Microbiol Antimicrob 5:2. doi:10.1186/14760711-5-2

7. Moran GJ, Krishnadasan A, Gorwitz RJ, EMERGEncy ID Net Study Group et al (2006) Methicillin-resistant $S$. aureus infections among patients in the emergency department. N Engl J Med 355:666-674. doi:10.1056/NEJMoa055356

8. Fowler VG Jr, Boucher HW, Corey GR et al (2006) Daptomycin versus standard therapy for bacteremia and endocarditis caused by Staphylococcus aureus. N Engl J Med 355:653-665. doi:10.1056/ NEJMoa053783
9. Smith TL, Pearson ML, Wilcox KR et al (1999) Emergence of vancomycin resistance in Staphylococcus aureus. N Engl J Med 340:493-501. doi:10.1056/NEJM199902183400701

10. Nishimura S, Tsurumoto T, Yonekura A, Adachi K, Shindo H (2006) Antimicrobial susceptibility of Staphylococcus aureus and Staphylococcus epidermidis biofilms isolated from infected total hip arthroplasty cases. J Orthop Sci 11:46-50. doi:10.1007/ s00776-005-0968-7

11. Marra A, Dib-Hajj F, Lamb L et al (2007) Enterococcal virulence determinants may be involved in resistance to clinical therapy. Diagn Microbiol Infect Dis 58:59-65. doi:10.1016/j.diagmicrobio.2006.11.024

12. Weigel LM, Donlan RM, Shin DH et al (2007) High-level vancomycin-resistant Staphylococcus aureus isolates associated with a polymicrobial biofilm. Antimicrob Agents Chemother 51:231238. doi:10.1128/AAC.00576-06

13. Tomasz A, Albino A, Zanati E (1970) Multiple antibiotic resistance in a bacterium with suppressed autolytic system. Nature 227:138-140. doi:10.1038/227138a0

14. Tuomanen E, Durack DT, Tomasz A (1986) Antibiotic tolerance among clinical isolates of bacteria. Antimicrob Agents Chemother 30:521-527

15. Goldenberg DL (1998) Septic arthritis. Lancet 351:197-202. doi:10.1016/S0140-6736(97)09522-6

16. Mader JT, Shirtliff ME, Bergquist S, Calhoun JH (2000) Bone and joint infections in the elderly: practical treatment guidelines. Drugs Aging 16:67-80. doi:10.2165/00002512-200016010-00006

17. Youssef PP, York JR (1994) Septic arthritis: a second decade of experience. Aust N Z J Med 24:307-311

18. Gottlieb T, Atkins BL, Shaw DR (2002) Soft tissue, bone and joint infections. Med J Aust 176:609-615

19. Hamed KA, Tam JY, Prober CG (1996) Pharmacokinetic optimisation of the treatment of septic arthritis. Clin Pharmacokinet 21:156-163. doi:10.2165/00003088-199631020-00006

20. Lazzarini L, Lipsky BA, Mader JT (2005) Antibiotic treatment of osteomyelitis: what have we learned from 30 years of clinical trials. Int J Infect Dis 9:127-138. doi:10.1016/j.ijid.2004.09.009

21. Stengel D, Bauwens K, Sehouli J, Ekkernkamp A, Porzsolt F (2001) Systematic review and meta-analysis of antibiotic therapy for bone and joint infections. Lancet Infect Dis 1:175-188. doi:10.1016/S1473-3099(01)00094-9

22. Appelbaum PC (2006) MRSA - the tip of the iceberg. Clin Microbiol Infect 12(suppl 2):3-10. doi:10.1111/j.1469-0691.2006. 01402.x

23. Hiramatsu K (2001) Vancomycin-resistant Staphylococcus aureus: a new model of antibiotic resistance. Lancet Infect Dis 1:147-155. doi:10.1016/S1473-3099(01)00091-3

24. Weigel LM, Clewell DB, Gill SR et al (2003) Genetic analysis of a high level vancomycin-resistant isolate of Staphylococcus aureus. Science 28:1569-1571. doi:10.1126/science.1090956

25. Hiramatsu K, Hanaki H, Ino T, Yabuta K, Oguri T, Tenover FC (1997) Methicillin-resistant Staphylococcus aureus clinical strain with reduced vancomycin susceptibility. J Antimicrob Chemother 40:135-136. doi:10.1093/jac/40.1.135

26. Ward PB, Johnson PD, Grabsch EA, Mayall BC, Grayson ML (2001) Treatment failure due to methicillin-resistant Staphylococcus aureus (MRSA) with reduced susceptibility to vancomycin. Med J Aust 175:480-483

27. Darley E, MacGowan A (2004) Antibiotic treatment of Grampositive bone and joint infection. J Antimicrob Chemother 53:928-935. doi:10.1093/jac/dkh191

28. Henry N, Roues M, Whitesell A (1987) Treatment of methicillin resistant Staphylococcus aureus experimental osteomyelitis with ciprofloxacin or vancomycin alone or in combination with rifampicin. Am J Med 82(suppl 4A):73-75 
29. Package insert (2003) Vancocin (vancomycin). Eli Lilly and Co, Indianapolis

30. Cunha BA (2005) Methicillin-resistant Staphylococcus aureus: clinical manifestations and antimicrobial therapy. Clin Microbiol Infect 11(suppl 4):33-42. doi:10.1111/j.1469-0691.2005.01162.x

31. Graber CJ, Wong MK, Carleton HA, Perdreau-Remington F, Haller BL, Chambers HF (2007) Intermediate vancomycin susceptibility in a community-associated MRSA clone. Emerg Infect Dis 13:491-493

32. Sakoulas G, Moise-Broder PA, Schentag J et al (2004) Relationship of MIC and bactericidal activity to efficacy of vancomycin for treatment of methicillin-resistant Staphylococcus aureus bacteremia. J Clin Microbiol 42:2398-2402. doi:10.1128/ JCM.42.6.2398-2402.2004

33. Finney MS, Crank CW, Segreti J (2005) Use of daptomycin to treat drug-resistant Gram-positive bone and joint infections. Curr Med Res Opin 21:1923-1926. doi:10.1185/030079905X74961

34. Package insert (2003) Synercid (quinupristin/dalfopristin) Monarch Pharmaceuticals, Bristol

35. Bouanchaud DH (1997) In-vitro and in-vivo antibacterial activity of quinupristin/dalfopristin. J Antimicrob Chemother 39(suppl A):15-21. doi:10.1093/jac/39.suppl_1.15

36. Saleh-Mghir A, Ameur N, Muller-Serieys C et al (2002) Combination of quinupristin-dalfopristin (Synercid) and rifampin is highly synergistic in experimental Staphylococcus aureus joint prosthesis infection. Antimicrob Agents Chemother 46:11221124. doi:10.1128/AAC.46.4.1122-1124.2002

37. Drew RH, Perfect JR, Srinath L, Kurkimilis E, Dowzicky M, Talbot GH (2000) Treatment of methicillin-resistant Staphylococcus aureus infections with quinupristin-dalfopristin in patients intolerant of or failing prior therapy. For the Synercid EmergencyUse Study Group. J Antimicrob Chemother 46:775-784. doi:10.1093/jac/46.5.775

38. Bassetti M, Righi E, DiBiagio A, Rosso R, Beltrame A, Bassetti D (2005) Role of linezolid in the treatment of orthopedic infections. Expert Rev Anti Infect Ther 3:343-352. doi:10.1586/14787210. 3.3.343

39. Package insert (2007) Zyvox (linezolid). Pharmacia \& Upjohn Co Div of Pfizer Inc., New York

40. Senneville E, Legout L, Valette M et al (2006) Effectiveness and tolerability of prolonged linezolid treatment for chronic osteomyelitis: a retrospective study. Clin Ther 28:1155-1163. doi:10.1016/ j.clinthera.2006.08.001

41. Falagas ME, Siempos II, Papagelopoulos PJ, Vardakas KZ (2007) Linezolid for the treatment of adults with bone and joint infections. Int J Antimicrob Agents 29:233-239. doi:10.1016/j.ijantimicag.2006.08.030

42. Prystowsky J, Siddiqui F, Chosay J et al (2001) Resistance to linezolid: characterization of mutations in rRNA and comparison of their occurrences in vancomycin-resistant enterococci. Antimicrob Agents Chemother 45:2154-2156. doi:10.1128/ AAC.45.7.2154-2156.2001

43. Tsiodras S, Gold HS, Sakoulas G et al (2001) Linezolid resistance in a clinical isolate of Staphylococcus aureus. Lancet 358:207208. doi:10.1016/S0140-6736(01)05410-1

44. Yin LY, Lazzarini L, Li F, Stevens CM, Calhoun JH (2005) Comparative evaluation of tigecycline and vancomycin with and without rifampicin, in the treatment of methicillin resistant Staphylococcus aureus experimental osteomyelitis in a rabbit model. J Antimicrob Chemother 55:995-1002. doi:10.1093/jac/ dki109

45. Package insert (2007) Tygacil (tigecycline). Wyeth Pharmaceuticals, Philadelphia

46. Doan TL, Fung HB, Mehta D, Riska PF (2006) Tigecycline: a glycylcycline antimicrobial agent. Clin Ther 28:1079-1106. doi:10.1016/j.clinthera.2006.08.011
47. Woodworth JR, Nyhart EH Jr, Brier GL, Wolny JD, Black HR (1992) Single-dose pharmacokinetics and antibacterial activity of daptomycin, a new lipopeptide antibiotic, in healthy volunteers. Antimicrob Agents Chemother 36:318-325

48. Tedesco KL, Rybak MJ (2004) Daptomycin. Pharmacotherapy 24:41-57. doi:10.1592/phco.24.1.41.34802

49. Silverman JA, Oliver N, Andrew T, Li T (2001) Resistance studies with daptomycin. Antimicrob Agents Chemother 45:1799-1802. doi:10.1128/AAC.45.6.1799-1802.2001

50. Ammerlaan HS, Bonten MJ (2006) Daptomycin: graduation day. Clin Microbiol Infect 12(suppl 8):22-28. doi:10.1111/j.14690691.2006.01627.x

51. Rybak MJ, Hershberger E, Moldovan T et al (2000) In vitro activities of daptomycin, vancomycin, linezolid, and quinupristindalfopristin against staphylococci and enterococci, including vancomycin-intermediate and -resistant strains. Antimicrob Agents Chemother 44:1062-1066. doi:10.1128/AAC.44.4.10621066.2000

52. Pfaller MA, Sader HS, Jones RN (2007) Evaluation of the in vitro activity of daptomycin against 19615 clinical isolates of Grampositive cocci collected in North American hospitals (2002-2005). Diagn Microbiol Infect Dis 57:459-465. doi:10.1016/j.diagmicrobio.2006.10.007

53. Credito K, Lin G, Appelbaum PC (2007) Activity of daptomycin alone and in combination with rifampin and gentamicin against Staphylococcus aureus assessed by time-kill methodology. Antimicrob Agents Chemother 51:1504-1507. doi:10.1128/ AAC.01455-06

54. Rand KH, Houck HJ (2004) Synergy of daptomycin with oxacillin and other beta-lactams against methicillin-resistant Staphylococcus aureus. Antimicrob Agents Chemother 48:2871-2875. doi:10.1128/AAC.48.8.2871-2875.2004

55. Tally FP, DeBruin MF (2000) Development of daptomycin for Gram-positive infections. J Antimicrob Chemother 46:523-526. doi:10.1093/jac/46.4.523

56. Tally FP, Zeckel M, Wasilewski MM et al (1999) Daptomycin: a novel agent for Gram-positive infections. Expert Opin Investig Drugs 8:1223-1238. doi:10.1517/13543784.8.8.1223

57. Oleson FB Jr, Berman CL, Kirkpatrick JB, Regan KS, Lai JJ, Tally FP (2000) Once-daily dosing in dogs optimizes daptomycin safety. Antimicrob Agents Chemother 44:2948-2953. doi:10.1128/ AAC.44.11.2948-2953.2000

58. Alder J, Li T, Yu D et al (2003) Analysis of daptomycin efficacy and breakpoint standards in a murine model of Enterococcus faecalis and Enterococcus faecium renal infection. Antimicrob Agents Chemother 47:3561-3656. doi:10.1128/AAC.47.11.35613566.2003

59. Cha R, Grucz RG Jr, Rybak MJ (2003) Daptomycin dose-effect relationship against resistant gram-positive organisms. Antimicrob Agents Chemother 47:1598-1603. doi:10.1128/ AAC.47.5.1598-1603.2003

60. Dandekar PK, Tessier PR, Williams P, Nightingale CH, Nicolau DP (2003) Pharmacodynamic profile of daptomycin against Enterococcus species and methicillin-resistant Staphylococcus aureus in a murine thigh infection model. $\mathrm{J}$ Antimicrob Chemother 52:405-411. doi:10.1093/jac/dkg337

61. Kaatz GW, Seo SM, Reddy VN, Bailey EM, Rybak MJ (1990) Daptomycin compared with teicoplanin and vancomycin for therapy of experimental Staphylococcus aureus endocarditis. Antimicrob Agents Chemother 34:2081-2085

62. Louie A, Kaw P, Liu W, Jumbe N, Miller MH, Drusano GL (2001) Pharmacodynamics of daptomycin in a murine thigh model of Staphylococcus aureus infection. Antimicrob Agents Chemother 45:845-851. doi:10.1128/AAC.45.3.845-851.2001

63. Mader JT, Adams K (1989) Comparative evaluation of daptomycin (LY146032) and vancomycin in the treatment of experimental 
methicillin-resistant Staphylococcus aureus osteomyelitis in rabbits. Antimicrob Agents Chemother 33:689-692

64. Smith K, Cobbs G, Dill R, Lyon D, Graves A, Avent K (1990) Daptomycin versus vancomycin treatment for Staphylococcus aureus bacteremia in a murine model. Chemotherapy 36:428-434

65. Luu QN, Buxton TB, Nelson DR, Rissing JP (1989) Treatment of chronic experimental Staphylococcus aureus osteomyelitis with LY146032 and vancomycin. Eur J Clin Microbiol Infect Dis 8:562-563. doi:10.1007/BF01967482

66. Safdar N, Andes D, Craig WA (2004) In vivo pharmacodynamic activity of daptomycin. Antimicrob Agents Chemother 48:63-68. doi:10.1128/AAC.48.1.63-68.2004

67. Schaad HJ, Bento M, Lew DP, Vaudaux P (2006) Evaluation of high-dose daptomycin for therapy of experimental Staphylococcus aureus foreign body infection. BMC Infect Dis 116:74. doi:10.1186/1471-2334-6-74

68. Yin L-Y, Calhoun JH, Tongchuan LI et al (2001) Comparative efficacies of vancomycin and cubicin, a novel lipopeptide antibiotic, in the treatment of methicillin-resistant Staphylococcus aureus: studies with a rabbit model. Presented at 39th annual meeting of the Infectious Diseases Society of America, San Francisco, 25-28 October, Abstract 358

69. Boucher HW, Abrutyn E, Savor-Price C et al (2006) Outcomes with daptomycin vs. standard therapy for $\mathrm{S}$. aureus bone and joint infections. Presented at 44th Annual Meeting of the Infectious Diseases Society of America Meeting, Toronto, Ontario, October 12-15. Abstract 377

70. Balter L, Donovan B, North D et al (2006) Retrospective evaluation of daptomycin efficacy and safety in patients with osteomyelitis. Presented at 44th annual meeting of the Infectious Diseases Society of America Meeting, Toronto, ON, Canada, 12-15 October, Abstract 208

71. Antony SJ, Angelos E, Stratton CW (2006) Clinical experience with daptomycin in patients with orthopedic related infections. Infect Dis Clin Pract 14:144-149. doi:10.1097/01.idc.0000206 490.05422.df

72. Rao N, Regalla DM (2006) Uncertain efficacy of daptomycin for prosthetic joint infections: a prospective case series. Clin Orthop Relat Res 451:34-37. doi:10.1097/01.blo.0000224021.73163.61

73. Holtom PD, Zalavras CG, Lamp KC, Park N, Friedrich LV (2007) Clinical experience with daptomycin treatment of foot or ankle osteomyelitis: a preliminary study. Clin Orthop Relat Res 461:35-39

74. Burns C (2008) Long-term use of daptomycin for MRSA osteomyletis and joint infection. Scand J Infect Dis 40:183-186. doi:10.1080/00365540701633038

75. Lamp KC, Friedrich LV, Mendez-Vigo L, Russo R (2007) Clinical experience with daptomycin for the treatment of patients with osteomyelitis. Am J Med 120(10A):S13-S20. doi:10.1016/j.amjmed.2007.07.010

76. Joseph TN, Chen AL, Di Cesare PE (2003) Use of antibioticimpregnated cement in total joint arthroplasty. J Am Acad Orthop Surg 11:38-47

77. Hall EW, Rouse MS, Jacofsky DJ et al (2004) Release of daptomycin from polymethyl-methacralate beads in a continuous flow chamber. Diagn Microbiol Infect Dis 50:261-265. doi:10. 1016/j.diagmicrobio.2004.03.004

78. Perry AC, Rouse MS, Khaliq Y et al (2002) Antimicrobial release kinetics from polymethylmethacrylate in a novel continuous flow chamber. Clin Orthop Relat Res 403:49-53. doi:10.1097/ 00003086-200210000-00009

79. Webb ND, McCanless JD, Courtney HS, Bumgardner JD, Haggard WO (2008) Daptomycin eluted from calcium sulfate appears effective against staphylococcus. Clin Orthop Relat Res 466:1383-1387. doi:10.1007/s11999-008-0245-0

80. Vikram HR, Havill NL, Koeth LM, Boyce JM (2005) Clinical progression of methicillin resistant Staphylococcus aureus vertebral osteomyelitis associated with reduced susceptibility to daptomycin. J Clin Microbiol 43:5384-5387. doi:10.1128/JCM.43.10. 5384-5387.2005

81. Marty FM, Yeh WW, Wennerstein CB et al (2006) Emergence of a clinical daptomycin resistant Staphylococcus aureus isolate during treatment of methicillin resistant Staphylococcus aureus bacteremia and osteomyelitis. J Clin Microbiol 44:595-597. doi:10.1128/JCM.44.2.595-597.2006

82. Hayden MK, Rezai K, Hayes RA, Lolans K, Quinn JP, Weinstein RA (2005) Development of daptomycin resistance in vivo in methicillin resistant Staphylococcus aureus. J Clin Microbiol 43:52855287. doi:10.1128/JCM.43.10.5285-5287.2005

83. Cui L, Tominaga E, Neoh HM, Hiramatsu K (2006) Correlation between reduced daptomycin susceptibility and vancomycin resistance in vancomycin-intermediate Staphylococcus aureus. Antimicrob Agents Chemother 50:1079-1082. doi:10.1128/ AAC.50.3.1079-1082.2006

84. Tenover FC, Moellering RC Jr (2007) The rationale for revising the Clinical and Laboratory Standards Institute vancomycin minimal inhibitory concentration interpretive criteria for Staphylococcus aureus. Clin Infect Dis 44:1208-1215. doi:10.1086/513203

85. Wootton M, MacGowan AP, Walsh TR (2006) Comparative bactericidal activities of daptomycin and vancomycin against glycopeptide-intermediate Staphylococcus aureus (GISA) isolates. Antimicrob Agents Chemother 50:4195-4197. doi:10.1128/ AAC.00678-06

86. Sader HS, Jones RN (2006) The activity of daptomycin against wild-type Staphylococcus aureus and strains with reduced susceptibility to vancomycin. Clin Infect Dis 43:798-799. doi:10.1086/ 507109

87. Sader HS, Fritsche TR, Jones RN (2005) Antimicrobial activity of daptomycin tested against clinical strains of indicated species isolated in North American medical centers (2003). Diagn Microbiol Infect Dis 53:329-332. doi:10.1016/j.diagmicrobio.2005.07.001

88. Sakoulas G, Brown J, Lamp KC et al (2006) Efficacy and safety of daptomycin in patients treated for non-catheter-related bacteremia. In: Program and abstracts of the 46th interscience conference on antimicrobial agents and chemotherapy, San Francisco, 27-30 September, Abstract 1536

89. Mohan SS, McDermott BP, Cunha BA (2005) Methicillin-resistant Staphylococcus aureus prosthetic aortic valve endocarditis with paravalvular abscess treated with daptomycin. Heart Lung 34:69-71. doi:10.1016/j.hrtlng.2004.07.009

90. Boucher HW, Sakoulas G (2007) Perspectives on daptomycin resistance, with emphasis on resistance in Staphylococcus aureus. Clin Infect Dis 45:601-608. doi:10.1086/520655

91. Rehm SJ, Boucher H, Levine DP et al (2006) Daptomycin vs. vancomycin for treatment of methicillin-resistant Staphylococcus aureus bacteremia and endocarditis. In: Program and abstracts of the 44th annual meeting of the Infectious Diseases Society of America, Toronto, ON, Canada, 12-15 October, Abstract 139

92. Sakoulas G, Alder J, Thauvin-Eliopoulos C, Moellering RC, Eliopoulos GM (2006) Induction of daptomycin heterogeneous susceptibility in Staphylococcus aureus by exposure to vancomycin. Antimicrob Agents Chemother 50:1581-1585. doi:10.1128/ AAC.50.4.1581-1585.2006

93. Sader HS, Watters AA, Fritsche TR, Jones RN (2007) Daptomycin antimicrobial activity tested against methicillin-resistant staphylococci, vancomycin-resistant enterococci isolated in European medical centers (2005). BMC Infect Dis 7:29. doi:10.1186/1471-2334-7-29

94. Critchley IA, Blosser-Middleton RS, Jones ME, Thornsberry C, Sahm DF, Karlowsky JA (2003) Baseline study to determine in vitro activities of daptomycin against Gram-positive pathogens isolated in the United States in 2000-2001. Antimicrob Agents Chemother 47:1689-1693. doi:10.1128/AAC.47.5.1689-1693. 2003 